\title{
THE MAXIMUM IDEMPOTENT-SEPARATING CONGRUENCE ON A REGULAR SEMIGROUP
}

\author{
by JOHN MEAKIN \\ (Received 4th December 1971)
}

\section{Introduction}

It has been established by G. Lallement (3) that the set of idempotentseparating congruences on a regular semigroup $S$ coincides with the set $\Sigma(\mathscr{H})$ of congruences on $S$ which are contained in Green's equivalence $\mathscr{H}$ on $S$. In view of this and Lemma 10.3 of A. H. Clifford and G. B. Preston (1) it is obvious that the maximum idempotent-separating congruence on a regular semigroup $S$ is given by

$$
\mu=\left\{(a, b) \in S \times S:(s a t, s b t) \in \mathscr{H}, \forall s, t \in S^{1}\right\} .
$$

The expression (1) for $\mu$ suffers from two maladies: it provides us with no information about $\mu$ which is not immediately deducible from Lallement's theorem and it is clearly not the sort of expression which may be readily used to decide if two given elements $a$ and $b$ of $S$ are related under $\mu$.

In (2), J. M. Howie gave and alternative expression for $\mu$ in the case where $S$ is an inverse semigroup. He determined that the maximum idempotentseparating congruence on an inverse semigroup $S$ is given by

$$
\mu=\left\{(a, b) \in S \times S \mid a e a^{-1}=b e b^{-1} \text { for all idempotents } e \text { of } S\right\} .
$$

A slightly different expression for $\mu$ in the case where $S$ is an inverse semigroup was obtained by Lallement in (3). In (4), the author has developed a characterization of the maximum idempotent-separating congruence on an orthodox semigroup (a regular semigroup whose idempotents form a subsemigroup). This characterization is similar to Howie's characterization (2): in fact, if $S$ is an orthodox semigroup, then $\mu$ is given by

$$
\begin{aligned}
\mu= & \left\{(a, b) \in S \times S \mid \text { there are inverses } a^{\prime} \text { of } a \text { and } b^{\prime} \text { of } b \text { for which } a e a^{\prime}=b e b^{\prime}\right. \\
& \text { and } \left.a^{\prime} e a=b^{\prime} e b \text { for all idempotents } e \text { of } S\right\} .
\end{aligned}
$$

In this note we develop an analogous characterization of the maximum idempotent-separating congruence on a regular semigroup.

\section{Preliminaries}

We use the notation and terminology of A. H. Clifford and G. B. Preston (1). In addition we denote the set of idempotents of a semigroup $S$ by $E_{S}$ and the 
set of inverses of an element $a$ in a regular semigroup $S$ by $V(a)$. Recall that we can define a partial ordering on the $\mathscr{L}$-classes of a semigroup $S$ by

$$
L_{a} \leqq L_{b} \text { iff } S^{1} a \subseteq S^{1} b
$$

and we can define a partial ordering on the $\mathscr{R}$-classes of $S$ by

$$
R_{a} \leqq R_{b} \text { iff } a S^{1} \subseteq b S^{1} .
$$

We also note that if $e$ and $f$ are idempotents of $S$ then

$$
L_{e} \leqq L_{f} \text { iff } e f=e \text { and } R_{e} \leqq R_{f} \text { iff } f e=e .
$$

We now introduce the following notation: if $a$ is an element of the semigroup $S$ then we define

and

$$
E L(a)=\left\{e \in E_{S} \mid L_{e} \leqq L_{a}\right\}
$$

$$
E R(a)=\left\{e \in E_{S} \mid R_{e} \leqq R_{a}\right\} .
$$

We remark that if $S$ is regular then for any $a \in S, E L(a) \neq \square$ and $E R(a) \neq \square$.

\section{The characterization of $\mu$}

We now prove the following theorem.

Theorem 2.1. The maximum idempotent-separating congruence on a regular semigroup $S$ is given by:

$$
\begin{aligned}
\mu= & \left\{(a, b) \in S \times S \mid \text { there are inverses } a^{\prime} \text { of } a \text { and } b^{\prime} \text { of } b\right. \text { such that } \\
& \left.a e a^{\prime}=b e b^{\prime} \forall e \in E L(a) \cup E L(b), \text { and } a^{\prime} f a=b^{\prime} f b \forall f \in E R(a) \cup E R(b)\right\} .
\end{aligned}
$$

Proof. It is obvious that $\mu$ is reflexive and symmetric. To show that $\mu$ is transitive, we first show that $\mu$ is contained in Green's equivalence $\mathscr{H}$.

Let $(a, b) \in \mu$ and let $a^{\prime}, b^{\prime}$ be the corresponding inverses of $a, b$ respectively, as in the definition (9) of $\mu$.

Note that $a a^{\prime} \in R_{a}$, so $a^{\prime} a=a^{\prime}\left(a a^{\prime}\right) a=b^{\prime}\left(a a^{\prime}\right) b$ and similarly

Hence

$$
b^{\prime} b=b^{\prime}\left(b b^{\prime}\right) b=a^{\prime}\left(b b^{\prime}\right) a, a a^{\prime}=b\left(a^{\prime} a\right) b^{\prime} \text { and } b b^{\prime}=a\left(b^{\prime} b\right) a^{\prime} .
$$

$$
\begin{array}{rlrl}
a a^{\prime} & =a\left(a^{\prime} a\right) a^{\prime}=a\left(b^{\prime} a a^{\prime} b\right) a^{\prime} & \\
& =b\left(b^{\prime} a a^{\prime} b\right) b^{\prime} & & \\
& =\left(b b^{\prime}\right)\left(a a^{\prime}\right)\left(b b^{\prime}\right) . &
\end{array}
$$

Hence

and

$$
\left(b b^{\prime}\right)\left(a a^{\prime}\right)=\left(b b^{\prime}\right)\left(a a^{\prime}\right)\left(b b^{\prime}\right)=a a^{\prime}
$$

$$
\left(a a^{\prime}\right)\left(b b^{\prime}\right)=\left(b b^{\prime}\right)\left(a a^{\prime}\right)\left(b b^{\prime}\right)=a a^{\prime} .
$$

By symmetry, $b b^{\prime}=\left(b b^{\prime}\right)\left(a a^{\prime}\right)=\left(a a^{\prime}\right)\left(b b^{\prime}\right)$, and so $a a^{\prime}=b b^{\prime}$. Similarly, $a^{\prime} a=b^{\prime} b$, and so since $a a^{\prime}=b b^{\prime}$ and $a^{\prime} a=b^{\prime} b$, it follows that $(a, b) \in \mathscr{H}$, and so $\mu \subseteq \mathscr{H}$.

In the sequel, if $a^{\prime} \in V(a)$ and $(a, b) \in \mathscr{H}$, we let $b^{\prime}$ denote the inverse of $b$ which is $\mathscr{H}$-related to $a^{\prime}$. 
We now prove that the relation $\mu$ defined by (7) is transitive. Let $(a, b) \in \mu$ and $(b, c) \in \mu$. Then $a \mathscr{H} b \mathscr{H} c$ and there are inverses $a^{\prime}$ of $a, b^{\prime}$ and $b^{*}$ of $b$ and $c^{*}$ of $c$ such that $a e a^{\prime}=b e b^{\prime}, b e b^{*}=c e c^{*}, \forall e \in E L(a)=E L(b)=E L(c)$, and $a^{\prime} f a=b^{\prime} f b, b^{*} f b=c^{*} f c, \forall f \in E R(a)=E R(b)=E R(c)$. Then $a a^{\prime}=b b^{\prime}$, $a^{\prime} a=b^{\prime} b, b b^{*}=c c^{*}, b^{*} b=c^{*} c$, and there exists $a^{*} \in V(a)$ and $c^{\prime} \in V(c)$ such that $a a^{\prime}=c c^{\prime}, c^{\prime} c=a^{\prime} a, a a^{*}=c c^{*}$ and $a^{*} a=c^{*} c$. Then for each

we have

$$
e \in E L(a)=E L\left(a^{\prime} a\right)=E L(b)=E L\left(b^{\prime} b\right)=E L(c),
$$

$$
\begin{aligned}
a(e) a^{*} & =a\left(e a^{\prime} a\right) a^{*}=\left(a e a^{\prime}\right)\left(a a^{*}\right)=\left(a e a^{\prime}\right)\left(b b^{*}\right)=\left(b e b^{\prime}\right)\left(b b^{*}\right) \\
& =b\left(e b^{\prime} b\right) b^{*}=b e b^{*}=c e c^{*},
\end{aligned}
$$

and for each $f \in E R(a)=E R(b)=E R(c)=E R\left(a a^{\prime}\right)=E R\left(b b^{\prime}\right)$, we have

$$
\begin{aligned}
a^{*} f a & =a^{*}\left(a a^{\prime} f\right) a=\left(a^{*} a\right)\left(a^{\prime} f a\right)=\left(b^{*} b\right)\left(a^{\prime} f a\right)=\left(b^{*} b\right)\left(b^{\prime} f b\right) \\
& =b^{*}\left(b b^{\prime} f\right) b=b^{*} f b=c^{*} f c .
\end{aligned}
$$

Hence $(a, c) \in \mu$, and so $\mu$ is transitive.

To prove that $\mu$ is left compatible, we first show that if $(a, b) \in \mu$ then $(c a, c b) \in \mathscr{H}$ for each $c \in S$. We let $(a, b) \in \mu$ and $c \in S$ and choose $a^{\prime} \in V(a)$, $b^{\prime} \in V(b)$ in accordance with the definition of $\mu$. Note first that

$$
(a, b) \in \mu \subseteq \mathscr{H} \subseteq \mathscr{R},
$$

and $\mathscr{R}$ is a left congruence on $S$, so $(c a, c b) \in \mathscr{R}$.

Now $c a=(c a)(c a)^{\prime}(c a)$ (where $(c a)^{\prime}$ is some inverse of $c a$ )

$$
\begin{aligned}
& =(c a)\left(a^{\prime} a\right)(c a)^{\prime} c a\left(a^{\prime} a\right)=(c a)\left(b^{\prime} b\right)(c a)^{\prime} c a\left(b^{\prime} b\right) \\
& =c a b^{\prime}\left[b\left((c a)^{\prime}(c a)\right) b^{\prime}\right] b .
\end{aligned}
$$

But $(c a)^{\prime}(c a)=\left((c a)^{\prime} c\right) a \in S^{1} a$, so $(c a)^{\prime}(c a) \in E L(b)$, and it follows that $b\left((c a)^{\prime}(c a)\right) b^{\prime}=a\left((c a)^{\prime}(c a)\right) a^{\prime}$.

Hence

$$
\begin{aligned}
c a & =(c a)\left(b^{\prime} a\right)(c a)^{\prime} c\left(a a^{\prime}\right) b \\
& =(c a)\left(b^{\prime} a\right)(c a)^{\prime}(c b) \in S^{1} c b,
\end{aligned}
$$

and so $L_{c a} \leqq L_{c b}$. Similarly, $L_{c b} \leqq L_{c a}$, and thus $(c a, c b) \in \mathscr{L}$. It follows that $(c a, c b) \in \mathscr{H}$, as required. We remark that in view of this fact, if $(c a)^{\prime} \in V(c a)$, then $(c b)^{\prime}$ denotes the inverse of $c b$ which is $\mathscr{H}$ - equivalent to $(c a)^{\prime}$. We now proceed to the proof of the left compatibility of $\mu$. Let $(a, b) \in \mu$ and $c \in S$.

Let $e \in E L(c a)=E L(c b)$. We show that if $(c a)^{\prime} \in V(c a)$, then

$$
c a e(c a)^{\prime}=c b e(c b)^{\prime} \text {. }
$$

As usual, $a^{\prime}$ and $b^{\prime}$ denote the inverses of $a$ and $b$ respectively which appear in the definition of $\mu$.

Now $e \in E L(c a)=E L\left((c a)^{\prime} c a\right)$ and so $e(c a)^{\prime}(c a)=e$. Also,

$$
L_{(c a)^{\prime}(c a)} \leqq L_{a^{\prime} a}=L_{a}
$$

so $e a^{\prime} a=e$ and $L_{e} \leqq L_{a}$. 
Hence

$$
\begin{aligned}
c a e(c a)^{\prime} & =c a e\left(a^{\prime} a\right)(c a)^{\prime}=c\left(a e a^{\prime}\right) a(c a)^{\prime} \\
& =c\left(b e b^{\prime}\right) a(c a)^{\prime}=(c b) e b^{\prime} a(c a)^{\prime}(c a)(c a)^{\prime} \\
& =(c b) e b^{\prime} a(c a)^{\prime}(c b)(c b)^{\prime} \\
& =(c b) e b^{\prime} a(c a)^{\prime}(c b)\left(b^{\prime} b\right)(c b)^{\prime} \\
& =(c b) e b^{\prime}\left[a(c a)^{\prime}(c a) a^{\prime}\right] b(c b)^{\prime} \\
& \left.=(c b) e b^{\prime}\left[b(c a)^{\prime}(c a) b^{\prime}\right] b(c b)^{\prime}, \text { (because } L_{(c a)^{\prime} c a} \leqq L_{a}=L_{b}\right) \\
& =(c b)\left(e b^{\prime} b\right)(c a)^{\prime}(c a)\left(a^{\prime} a\right)(c b)^{\prime} \\
& =(c b) e(c b)^{\prime}(c b)(c b)^{\prime}=(c b) e(c b)^{\prime} .
\end{aligned}
$$

Now let $f \in E R(c a)=E R(c b)=E R\left((c a)(c a)^{\prime}\right)$. Then $(c a)(c a)^{\prime} f=f$ and it follows that $(c a)^{\prime} f(c a) \in E_{S}$ and that $(c a)^{\prime} f(c a) \in E L(a)=E L(b)$.

Hence,

$$
\begin{aligned}
(c a)^{\prime} f(c a) & =(c a)^{\prime}(c a)(c a)^{\prime} f(c a)(c a)^{\prime}(c a) \\
& =(c b)^{\prime}(c b)\left[(c a)^{\prime} f(c a)\right](c b)^{\prime}(c b) \\
& =(c b)^{\prime}(c b)\left(b^{\prime} b\right)\left[(c a)^{\prime} f(c a)\right]\left(a^{\prime} a\right)(c b)^{\prime}(c b) \\
& =(c b)^{\prime}\left(c b b^{\prime}\right) b\left[(c a)^{\prime} f(c a)\right]\left(b^{\prime} b\right)(c b)^{\prime}(c b) \\
& =(c b)^{\prime}\left(c b b^{\prime}\right) a\left[(c a)^{\prime} f(c a)\right] a^{\prime} b(c b)^{\prime}(c b) \\
& =(c b)^{\prime}(c a)\left(a^{\prime} a\right)\left[(c a)^{\prime} f(c a)\right] a^{\prime} b(c b)^{\prime}(c b) \\
& =(c b)^{\prime}(c a)(c a)^{\prime} f c\left(a a^{\prime}\right) b(c b)^{\prime}(c b) \\
& =(c b)^{\prime}(c a)(c a)^{\prime} f(c b)(c b)^{\prime}(c b) \\
& =(c b)^{\prime}(c b)(c b)^{\prime} f(c b)(c b)^{\prime}(c b)=(c b)^{\prime} f(c b)
\end{aligned}
$$

Thus $\mu$ is left compatible: a similar argument shows that $\mu$ is right compatible, and so $\mu$ is a congruence. Since $\mu \subseteq \mathscr{H}, \mu$ is an idempotent-separating congruence.

Finally, let $\rho$ be any idempotent-separating congruence on $S$, and let $(a, b) \in \rho$. Then $(a, b) \in \mathscr{H}$, and so there are inverses $a^{\prime}$ of $a$ and $b^{\prime}$ of $b$ such that $a a^{\prime}=b b^{\prime}$ and $a^{\prime} a=b^{\prime} b$.

Let $e \in E L(a)=E L\left(a^{\prime} a\right)=E L\left(b^{\prime} b\right)=E L(b)$. Then $e a^{\prime} a=e=e b^{\prime} b$, and $\left(a e a^{\prime}\right)\left(a e a^{\prime}\right)=a\left(e a^{\prime} a\right) e a^{\prime}=a e e a^{\prime}=a e a^{\prime} \in E_{S}$, and similarly $b e b^{\prime} \in E_{S}$. Also, $b^{\prime}=b^{\prime} b b^{\prime}=b^{\prime} a a^{\prime} \rho b^{\prime} b a^{\prime}=a^{\prime} a a^{\prime}=a^{\prime}$, so $\left(a^{\prime}, b^{\prime}\right) \in \rho$, and hence $\left(a e a^{\prime}, b e b^{\prime}\right) \in \rho$. Since $a e a^{\prime}, b e b^{\prime} \in E_{\mathrm{S}}$, this implies that $a e a^{\prime}=b e b^{\prime}$. Similarly, $a^{\prime} f a=b^{\prime} f b$ for each $f \in E R(a)=E R(b)$, and so $(a, b) \in \mu$. Thus $\rho \subseteq \mu$ and this completes the proof that $\mu$ is the maximum idempotent-separating congruence on $S$.

\section{REFERENCES}

(1) A. H. Cllfford and G. B. Preston, The Algebraic Theory of Semigroups (Math. Surveys, No. 7, Amer. Math. Soc., Vol. I, 1961, Vol II, 1967). 
THE MAXIMUM IDEMPOTENT-SEPARATING CONGRUENCE 163

(2) J. M. HowrE, The maximum idempotent-separating congruence on an inverse semigroup, Proc. Edinburgh Math. Soc. (2) 14 (1964-65), 71-79.

(3) G. Lallement, Demi-groupes réguliers, Ann. Mat. Pura Appl. (4) 77 (1967), 47-129.

(4) J. C. Meakin, Congruences on orthodox semigroups, J. Austral. Math. Soc. 12 (1971), 323-341.

ThE UNIVERSITY OF Nebraska

LiNCOLN, NEBRASKa 68508,

U.S.A. 